\title{
Preventive Effect of Cinnamon Oil Against Diethylnitrosamine-Induced Hepatocellular Carcinoma in Albino Rats: Histopathological and Serum Biochemical Evaluation
}

\author{
Aly S. M., Fetaih H. A. and Aliaa A. Ismail \\ Department of Pathology, Faculty of veterinary Medicine, Suez Canal \\ University, Ismailia, Egypt.
}

\begin{abstract}
Oxidative stress has been implicated in pathogenesis of various disease events including cancer, cardio vascular diseases, immune system disorders, brain dysfunction and metabolic diseases. The present study aimed to evaluate the protective effect of cinnamon oil on diethylnitrosamine (DENA) and 2-acetylaminoflourene (2-AAF) induced hepatocarcinogenesis. Thirty male albino rats were classified into 3 equal groups. Group (A), rats were kept as control. Group (B), rats received single intraperitoneal injection of DENA (200 mg/kg b.wt.) and 2-AAF ( $0.03 \% \mathrm{w} / \mathrm{w}$ of diet for 10 successive weeks). Group (C), rats received cinnamon oil $100 \mathrm{mg} / \mathrm{kg}$ b.wt. orally via stomach tube daily one week prior to HCC induction (that occurred same way as group B at $2^{\text {nd }}$ week). Rats were sacrificed at 3 months of the experiment. HCC-induced group (B) showed significant decrease in body mass and significant elevation in liver weight, alpha-fetoprotein (AFP), liver enzymes as well as hepatic malondialdehyde (MDA). In addition to the decrease of Glutathione (GSH) level and superoxide dismutase (SOD) activities. While, HCC rats -pretreated with cinnamon oil in group $\mathrm{C}$, were significantly reversed these destructive actions, with reduced liver toxicity. Histopathologically, the liver of HCC-induced group (B) showed cancer features including hypercellularity, nuclear hyperchromatia and miototic figures. HCC rats pretreated with cinnamon oil revealed partial reversal to the normal liver architecture. The present findings proposed that, this natural oil has the ability to improve liver function, significantly reduced the liver toxicity and HCC development. However, further investigations are needed before wide scale recommendation.
\end{abstract}

Key words: Hepatocellular carcinoma, male Albino rats, Diethylnitrosamine, 2-Acetylaminoflourene, cinnamon oil.

\section{Introduction}

Hepatocellular carcinoma (HCC) is one of the most prevalent and well- known malignancies. It is the second most leading cause of cancer death and accounts for $70-85 \%$ of 
the primary malignant tumors of the liver (Rouleau et al., 2016). Prominent risk factors associated with the development of HCC include cihrrosis, ingestion of aflatoxin B1-contaminated food, chronic alcohol consumption and hepatitis B as well as $\mathrm{C}$ viral infections. A wide variety of treatment options for HCC, including chemotherapy, surgical approaches, Ablation, Transarterial chemoembolization and Systemic therapy (Sorafenib), showed limited benefits, harmful side effects and complications (Kew, 2014). In this context, attempts have been made to find out a better treatment compounds that will improve the overall prognosis of HCC and selectively target and block the tumor-specific pathways in HCC. Regarding this, naturally occurring polyphenols are receiving increased attention because of natural products are believed to suppress the inflammatory process that lead to neoplastic transformation, hyperproliferation, promotion and progression of carcinogenic process and angiogenesis (Mnayer et al., 2014).

DENA is a potent hepatocarcinogenic chemical and the most widely used initiator for $\mathrm{HCC}$ induction in animal models. It is one of N-nitroso compounds which are serious health hazards (Santos et al., 2017). DENA is metabolized, by cytochrome P450 in liver, into the pro-mutagenic adducts: O6- ethyleoxyguanosine, as well as 04, O6-ethyldeoxythymidine leading to cytotoxicity, mutagenicity and carcinogenicity.

2-AAF is one of the peroxisome proliferators which show promoting activity on rat liver cancer (De Minicis et al., 2013). 2-AAF induces increase in reactive oxygen species (ROS) leading to oxidative stress. ROS are highly cytotoxic agents that lead to damage to all major biological macromolecules thereby leading to mis-repair of DNA damage and lipid peroxidation resulting in mutations such as base substitution and deletion which lead to carcinogenesis (Hassan et al., 2016).

Cinnamon, belongs to the family Lauraceae, is widely used in traditional medicine and as a flavouring agent (Qin et al., 2014). Cinnamon possesses significant anticancer, antibacterial, antifungal, insulin potentiating, antioxidant and anti-inflammatory properties (Wong et al., 2014).

Cinnamon exerts it's antitumor activity through increasing the glutathione $\mathrm{S}$-transferase (GST) activity resulting in free radicals elimination (Anderson et al., 2016) that reducing the tumor cell growth and induction of apoptosis (Sariözkan et al., 2016).

The present study aimed to investigate the protective effect of cinnamon oil on diethylnitrosamine (DENA) and 2-acetylaminoflourene 
(2-AAF) induced hepatocarcinogenesis through histopathological and serum biochemical examinations.

\section{Material and Methods \\ Experimental Animals:}

Pathogen-free apparent healthy 30 male albino rats weighing $(180 \pm 10$ g) obtained from the Animal House of Theodor Bilharz Research Institute were used in the present study. All animals were housed in the Laboratory Animal House, Faculty of Veterinary Medicine, Suez Canal University, Ismailia, Egypt under standard environmental temperature $\left(23 \pm 2^{\circ} \mathrm{C}\right)$ with 12-hour light/dark cycle in a well-ventilated room with free access to commercial standard diet and tap drinking water.

Carcinogenic chemicals and hepatoprotective oil:

Diehylnitrosamine (DENA) and 2acetylaminofluorene (2-AAF) were purchased from Sigma-Aldrich Chemicals Co. (St. Louis, MO, USA). Cinnamon oil was purchased from Captain Natural Oils Company, Cairo, Egypt. It was administered orally by stomach tube at a dose of $100 \mathrm{mg} / \mathrm{Kg}$ B.wt. modified after Yüce et al., (2014).

\section{Chemical kits:}

Serum alpha-fetoprotein (AFP) concentrations were estimated using kits obtained from Kamiya Biomedical Company, USA. Serum biochemical parameters were performed using ready-made kits to determine liver enzymes obtained from (Randox Co.UK). Readymade kits for reduced glutathione (GSH), superoxide dismutase (SOD) and malondialdehyde (MDA) were purchased from biodiagnostics, Cairo, Egypt.

\section{Induction of hepatocellular carcinoma:}

Hepatocarcinogenesis was induced, using tumor initiation and promotion model. Initiation was induced by single intraperitoneal (i.p) injection of DENA at a dose of $200 \mathrm{mg} / \mathrm{kg}$ body wt. diluted in normal saline. The animals were not given any further treatment for 2 weeks. After this, HCC development was promoted by feeding the animals a diet containing 2-AAF at a dose of $0.03 \% \mathrm{w} / \mathrm{w}$ of diet for 10 successive weeks (Abdel-Hamid et al., 2017).

\section{Experimental design:}

Group A: Normal control: served as negative control rats. Ten rats were orally administered $1 \mathrm{ml}$ corn oil/rat for the entire period of the experiment.

Group B: HCC-induced: treated with DENA and 2-AAF without any other additional treatment.

Group C: Cinnamon oil HCCpretreated group: Rats were pretreated orally with cinnamon oil $(100 \mathrm{mg} / \mathrm{Kg}$ BW) daily for 7 days before HCC induction (MUHAMMAD et al., 2017). Oral administration of cinnamon oil continued along with concomitant administration of 2-AAF in the diet for the duration of the experiment. 


\section{Sampling:}

After 13 weeks, rats were subjected to mild ether anesthesia and blood samples were collected. Serum was separated and stored at $-20{ }^{\circ} \mathrm{C}$ to be used for AFP levels detection and biochemical analysis. After blood collection, rats were dissected and specimens from internal organs (liver, lung, intestine and kidney) were taken. The livers were excised, weighed and small sections were cut. The first liver section was used for the preparation of the liver tissue homogenate to be used for the antioxidant state analysis. The second liver section was fixed in $10 \%$ neutral buffered formalin for the histopathological examination.

Determination of tumor marker level Alpha-fetoprotein (AFP) in rat serum:

AFP level in serum of rat blood was determined at $450 \mathrm{~nm}$ by using enzyme linked immunosorbent assay (ELISA) test kit. Determination of liver function enzymes:

Serum parameters as ALT, AST, ALP and GGT were measured using respective kits according to the manufacturer's instructions.

Determination of Antioxidant status and lipid peroxidation in liver

The hepatic GSH content, SOD activity and lipid peroxidation were estimated according to the method of Elguindy et al., (2016).

\section{Histopathological Examination:}

Specimens of liver, lung, intestine and kidney were freshly taken and fixed in $10 \%$ neutral buffered formalin, then washed in running tap water, dehydrated in ascending concentration gradients of ethyl alcohol and cleared in two changes of xylene then embedded in paraffin wax and afterwards sliced into thin sections of $5 \mu \mathrm{m}$ thickness using rotatory microtome. Sections were stained with $H \& E$ stain according to the standard method (Patial et al., 2015) then examined microscopically.

\section{Statistical analysis}

Statistical analyses were performed using software SPSS version 20.0. One-way analysis of variance (ANOVA) was used to detect the significant changes between the groups followed by Duncan's multiple comparison test to find if there was any

significant difference between groups. Statistical significance was considered when $P \leq 0.05$. All the values were expressed as mean \pm SE (standard error of the mean).

\section{Results}

Changes in Final body weight and absolute liver weight:

A high significant decrease in body weight of HCC-induced rats was observed compared to the control ones. Moreover, liver weight of HCC-induced group showed significant increase compared with the control group. There was a significant increase in the body 
weight and significant decrease in liver weight of cinnamon oil HCCpretreated animals compared with HCC-induced group.

Tumor Marker Enzyme (AFP), Serum biochemical analyses, liver oxidative Stress and lipid peroxidation (MDA):

In general, the levels of AFP, liver enzymes (ALT, AST, ALP and GGT) and MDA were significantly increased in $\mathrm{HCC}$-induced group compared to the control one. In addition, antioxidant enzymes (GSH and SOD) were significantly decreased in HCC-induced rats compared to the control ones. On the other hand, cinnamon oil HCCpretreated group showed significant decrease in AFP, liver enzymes and MDA levels in comparison with HCC-induced group. Moreover, GSH and SOD levels were significantly increased in cinnamon oil HCC-pretreated group compared to HCC-induced group (Figs. 1, 2, 3 and 4).

\section{Pathological results:}

\section{Gross pathology:}

Rats of control group showed normal shape and size of organs. No gross lesions observed in all organs and the liver exhibited a smooth, glossy surface and a soft texture. In HCC-induced group, gross liver changes were moderate enlargement and swelling of liver with rough and rounded edges, increased friability of the parenchyma, dark red color and slightly smooth surface. No particular changes were noted in other organs. Cinnamon oil HCC- pretreated group showed almost normal appearance with slight enlargement of liver. Other organs were of normal shape and color.

Histopathological examination: Histopathological examination of the liver:

The histological examination of liver from control rats revealed normal hexagonal lobular pattern of hepatic tissue with normal size and shape of hepatocytes. In HCCinduced group, the neoplastic changes included massive increase in cell density. Also, the nuclei of many hepatocytes were enlarged, prominent and pleomorphic. There was an increase in nuclear chromatin and irregular chomatin distribution within nuclei (triple or quadruple nuclear parts) with hyperchromatism. Also, multinucleation or binucleation (frequent mitoses) was evident in large number of cells (welldifferentiated HCC). In Cinnamon oil HCC-pretreated group, liver tissues showed slight increase in cell number. Hepatocytes were mildly enlarged and showed little heteromorphism. The nuclei increased in size, hyperchromatic, pleomorphic and binucleolation was observed (Fig. 5).

Histopathological examination of other internal organs:

The internal organs (Intestine, Kidney, Lung) in rats of control group did not show any histopathological alterations.The intestine from $\mathrm{HCC}$-induced rats showed moderate to severe 
catarrhal enteritis. HCC-induced rats and pretreated with cinnamon oil showed mild mucinous degeneration, slight desquamation of intestinal epithelium and some leukocytic infiltration in lamina propria submucosa (Fig. 6). Kidnies from HCC-induced rats showed tubular nephrosis, over dilated blood vessels and numerous renal casts. Cinnamon oil HCC-pretreated group showed mild congestion of the intertubular blood capillaries, some inflammatory cells infiltration

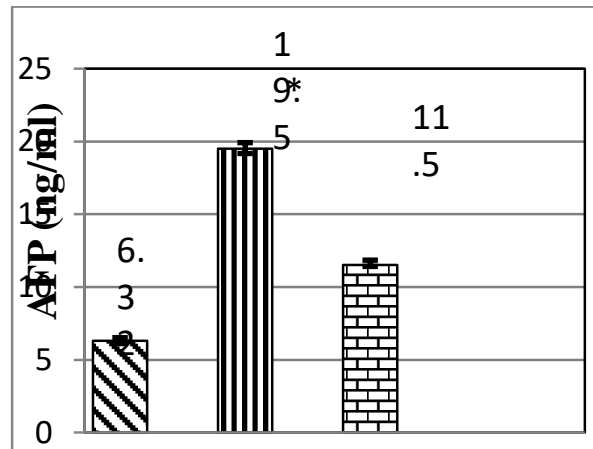

Control AGramon oil-HCC

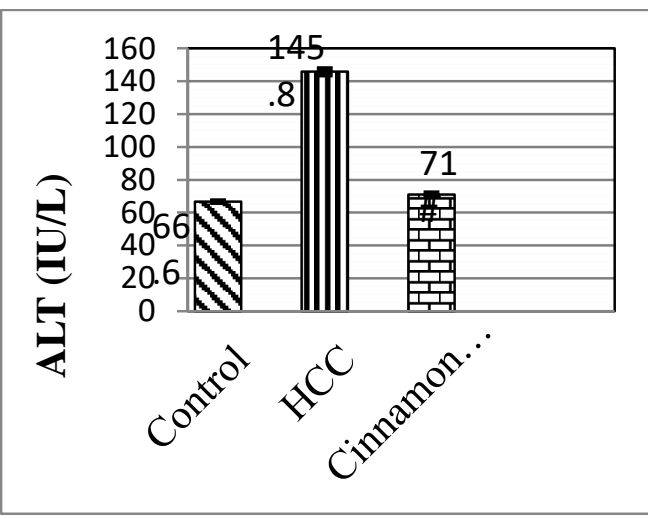

in renal tissue. The lungs from HCC-induced rats showed moderate congestion of inter alveolar and perbronchial blood capillaries, moderate perbronchial leukocytic infiltration, slight desqumation of bronchial epithelium. Cinnamon oil HCC-pretreated group showed slight thickening of alveolar walls with inflammatory cells (Fig. 7).

Fig. 1: AFP levels in serum of experimented rats. Values are expressed as mean \pm SE $(p \leq 0.05$ was considered significant): * for diseased vs. control, and \# for diseased vs. pretreated.

Fig. 2: Serum ALT levels of experimented rats. Values are expressed as mean $\pm \mathrm{SE}(\mathrm{p} \leq 0.05$ was considered significant): * for diseased vs. control, and \# for diseased vs. pretreated. 


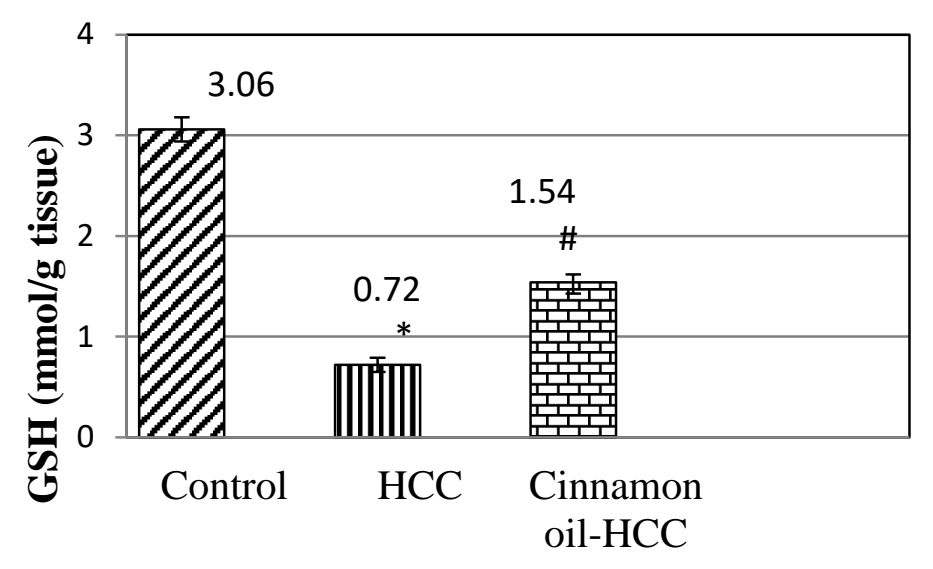

Fig. 3: GSH levels of rats in different experimented groups. Values are expressed as mean $\pm \mathrm{SE}$ ( $\mathrm{p} \leq 0.05$ was considered significant): * for diseased vs. control, and \# for diseased vs. pretreated.

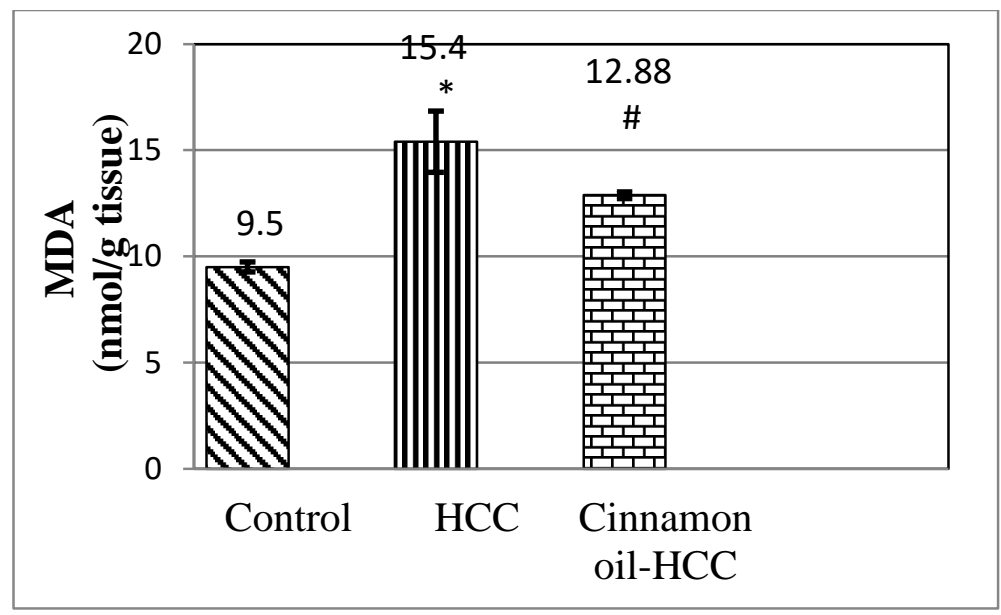

Fig. 4: MDA levels of rats in different experimented groups. Values are expressed as mean $\pm \mathrm{SE}(\mathrm{p} \leq 0.05$ was considered significant): * for diseased vs. control, and \# for diseased vs. pretreated. 

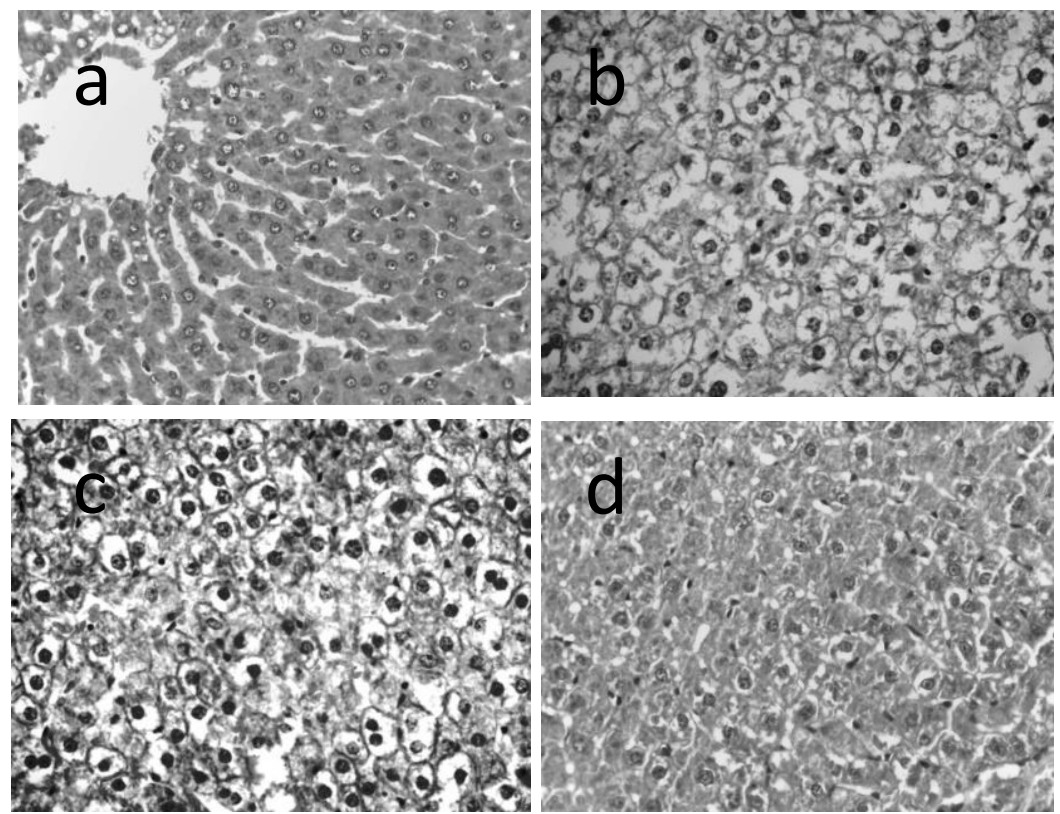

Fig. 5. Liver tissue sections in control (a) and experimental rats: HCCinduced group showing prominent, pleomorphic and hyperchromatic nuclei (b). Cinnamon oil-HCC pretreated group image showing nearly restoration of liver cell architecture (c). H\&E stain, X100.
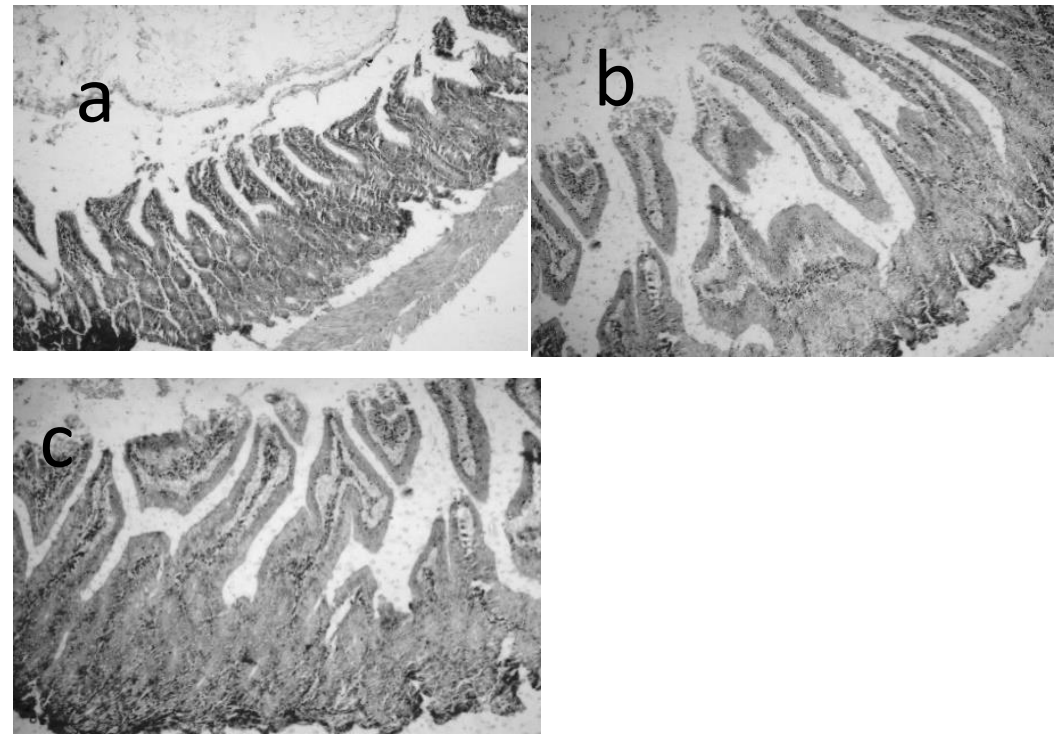

Fig. 6. Intestine in control (a) and experimental rats. HCC-induced group showing moderate mucinous degeneration and mild necrosis of tips of the 
intestinal villi (b). Cinnamon oil-HCC pretreated group image showing mild desquamation of intestinal epithelium (c). H\&E stain, X100.
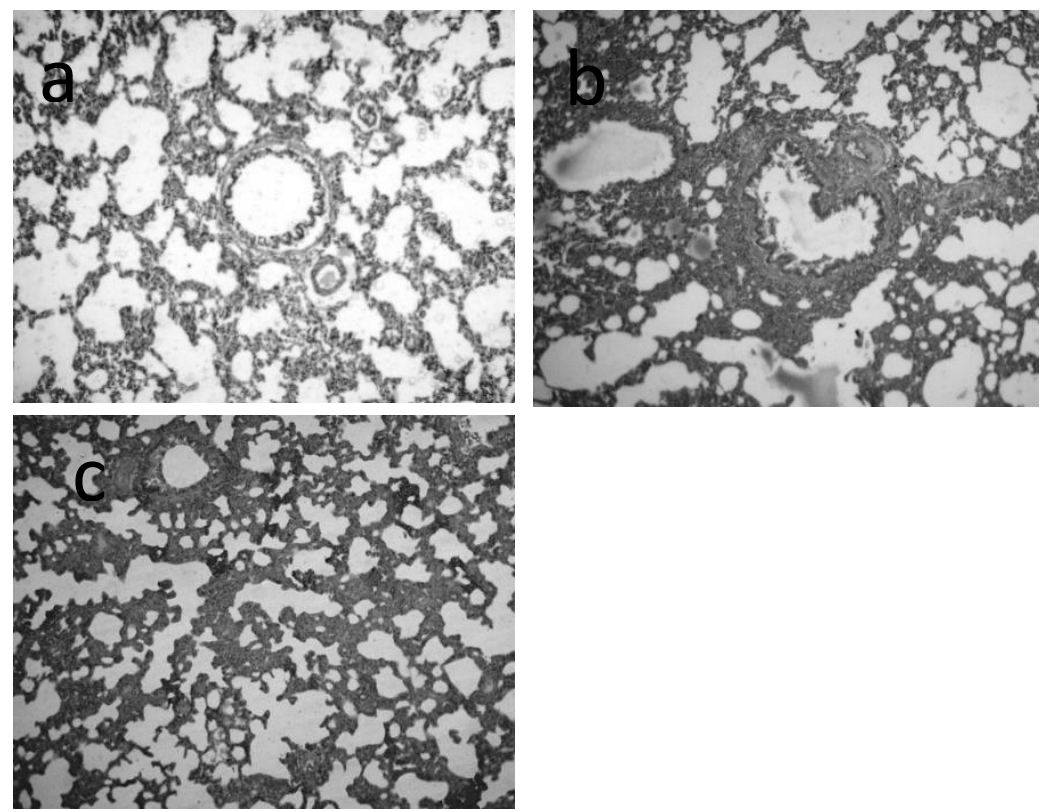

Fig. 7. Lung of control (a) and experimental rats. HCC-induced group showing mild perbronchial leukocytic infiltration (b). Cinnamon oil-HCC pretreated group image showing slight thickening of alveolar walls with inflammatory cells (c). H\&E stain, X100.

\section{Discussion}

Liver plays a vital role in neutralization of exo and endotoxins (Niu et al., 2016). The limited treatment options and poor treatment success make HCC one of the leading causes of death in developing countries. Therefore, evaluation of new active chemotherapeutic agents is an urgent need to improve the survival of patients with advanced or recurrent HCC (Weerapreeyakul et al., 2016).
Final body weight and absolute liver weight:

In this study, there was a progressive loss in body weight of HCC-induced rats throughout the experiment duration and this reduction in body weight correlated with decreased food intake. Loss of appetite, inadequate food intake and impaired digestion and absorption may contribute to this weight loss (Schneider et al., 2017).

The steadily increase in body weight of cinnamon oil HCCpretreated group may resulted from increased appetite and food consumption, improved intestinal 
villi structure and increased digestion and absorption.

Concerning the increase in absolute liver weight that observed in rats with HCC can be potentially attributed to hepatocytes hyperplasia and hypertrophy. However, cinnamon oil was found to reduce the absolute liver weight that showing it's anticancer effectiveness as proved through the histopathological findings.

Tumor Marker Enzyme (AFP):

AFP levels were significantly raised in HCC-induced rats, which may have resulted from hepatic intoxication that caused increased transcription of AFP gene.

Serum biochemical analyses:

Regarding the serum biochemical analyses, hepatocellular damage caused by DENA and 2-AAF leads to distinctive increase in the serum enzyme activities (Faloppi et al., 2016). On the other hand, cinnamon oil pretreatment to HCC rats significantly lowered the enhanced levels of these enzymes. This might be due to the presence of hepatoprotective natural bioactive constituents in the oily extract of cinnamon, which have the ability to reduce free radical-induced hepatic damage as observed in the current histopathological results.

\section{Liver oxidative Stress:}

GSH and SOD levels were decreased in HCC-induced animals. The depletion in activity of these enzymes may be caused by the increase in free radicals generation from metabolism of carcinogenic chemicals used (Hassan et al., 2016). In cinnamon oil HCCpretreated rats, higher levels of hepatic GSH and SOD may be due to suppression of lipid peroxidation (Sathya et al., 2014).

MDA increased in HCC-induced rats indicating high level of lipid peroxidation and failure of antioxidant defense mechanisms. However, cinnamon oil HCCpretreated group displayed a significant reduction in MDA level. This could be presumably related to it's ability to scavenge reactive oxygen species.

\section{Histopathological examination:}

DENA is known to cause enlargement of the nucleus of hepatocytes, decreased glycogen content and centrilobular necrosis. Nuclear changes explain the presence of different cell populations, which occur during DNA replication and cell division phases. The increase in nuclear chromatin is due to increased DNA content. Nevertheless, pretreatment with cinnamon oil to $\mathrm{HCC}$ rats not only inhibited the adverse effects of DENA/2-AAF but also helped in partial improvement of histological features of liver.

\section{Conclusion}

This study substantiates the biochemical, antioxidant and pathophysiological preventive properties of cinnamon oil (100 $\mathrm{mg} / \mathrm{kg} \mathrm{BW}$ ) as anti-hepatocellular carcinoma agent. Taken together all 
the results suggest that regular use of cinnamon oil may be attractive and useful therapeutic approach to control proliferation of liver cancer cells, however, further investigations are needed before wide scale recommendation.

\section{References}

Anderson, R.A., Zhan, Z., Luo, R., Guo, X., Guo, Q., Zhou, J., Kong, J., Davis, P.A. \& Stoecker, B.J. (2016): Cinnamon extract lowers glucose, insulin and cholesterol in people with elevated serum glucose. Journal of traditional and complementary medicine.336-332,6,

De Minicis, S., Kisseleva, T., Francis, H., Baroni, G.S., Benedetti, A., Brenner, D., Alvaro, D., Alpini, G. \& Marzioni, M. (2013): Liver carcinogenesis: rodent models of hepatocarcinoma and cholangiocarcinoma. Digestive and Liver Disease, 45.459-450,

Hassan, H.A., El-Gharib, N.E. \& Azhari, A.F. (2016): Role of natural antioxidants in the therapeutic management of hepatocellular carcinoma. Hepatoma Res, 2, 216-223.

Kew, M.C. 2014. Hepatocellular carcinoma: epidemiology and risk factors. Journal of hepatocellular carcinoma, 1, 115.

Mnayer, D., Fabiano-Tixier, A.S., Petitcolas, E., Hamieh, T., Nehme, N., Ferrant, C., Fernandez, X. \& Chemat, F. (2014): Chemical composition, antibacterial and antioxidant activities of six essentials oils from the Alliaceae family. Molecules, 19, 20034-20053.

MUHAMMAD, A., TAHIR, M. \& NASEEM, N. (2017): EFFECT OF CINNAMON BARK OIL ON CADMIUM INDUCED TESTICULAR TOXICITY IN MALE ALBINO RATS. Biomedica, 33.

Niu, J., Lin, Y., Guo, Z., Niu, M. \& Su, C. (2016): The Epidemiological investigation on the risk factors of hepatocellular carcinoma: a case-control study in Southeast China. Medicine, 95.

Qin, B., Panickar, K.S. \& Anderson, R.A. (2014): Cinnamon polyphenols attenuate the hydrogen peroxide-induced down regulation of $S 100 \beta$ secretion by regulating sirtuin 1 in C6 rat glioma cells. Life sciences, 102, 72-79.

Rouleau, L., Antony, A.N., Bisetto, S., Newberg, A., Doria, C., Levine, M., Monti, D.A. \& Hoek, J.B. (2016): Synergistic effects of ascorbate and sorafenib in hepatocellular carcinoma: New insights into ascorbate cytotoxicity. Free Radical Biology and Medicine, 95, 308-322.

Santos, N.P., Colaço, A.A. \& Oliveira, P.A. (2017): Animal models as a tool in hepatocellular carcinoma research: A Review. Tumor Biology, 39, 10.10428317695923

Sariözkan, S., Türk, G., Güvenç, M., Yüce, A., Özdamar, S., Cantürk, F. \& Yay, A.H. (2016): Effects of cinnamon (C. 
zeylanicum) bark oil against taxanes-induced damages in sperm quality, testicular and epididymal oxidant/antioxidant balance, testicular apoptosis, and sperm DNA integrity. Nutrition and cancer, 68, 481-494.

Sathya, M., Rajeshwari, V. \& Sakthishree, K. (2014): Influence of aqueous extract of Cinnamomum zeylanicum on the progression of cancer in diethylnitrosamine induced rat liver. European Journal of Experimental Biology, 4, 190195.

Schneider, A.T., Gautheron, J., Feoktistova, M., Roderburg, C., Loosen, S.H., Roy, S., Benz, F., Schemmer, P., Büchler, M.W. \& Nachbur, U. (2017): RIPK1 suppresses a TRAF2-dependent pathway to liver cancer. Cancer Cell, 31, 94-109.
sWeerapreeyakul, N., Junhom, C., Barusrux, S. \& Thitimetharoch, T. (2016): Induction of apoptosis in human hepatocellular carcinoma cells by extracts of Lannea coromandelica (Houtt.) Merr. and Diospyros castanea (Craib) Fletcher. Chinese medicine, 11, 19.

Wong, Y., Ahmad-Mudzaqqir, M. \& Wan-Nurdiyana, W. (2014): Extraction of essential oil from cinnamon (Cinnamomum zeylanicum). Oriental journal of chemistry, 30, 37-47. 


\title{
الملخص باللغة العربية
}

التأثير الوقائى لزيت القرفة على سرطان الخلايا الكبدية المستحدث كيمائيا بإستخدام

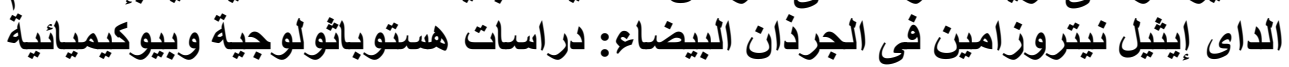

\author{
صلاح الدين مصيلحى على - أحمدى عبدالوهاب فتيح ـعلياء عبدالرحيم أحمد اسماعيل
}

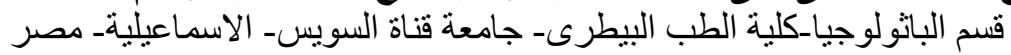

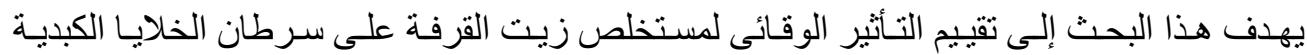

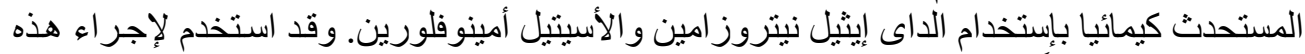

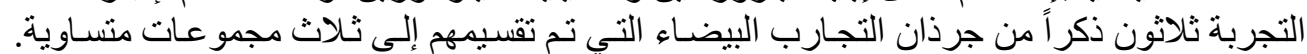

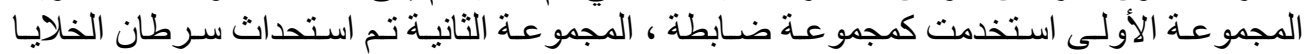

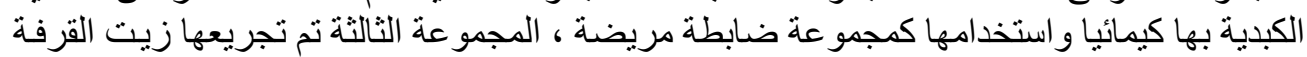

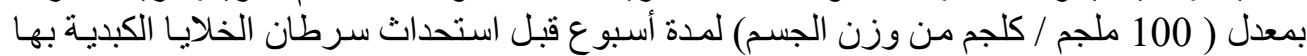

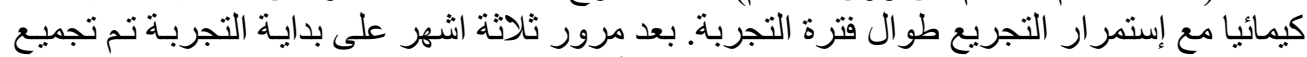

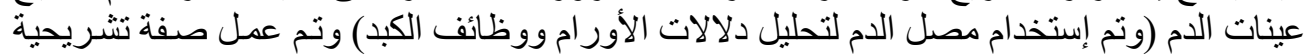

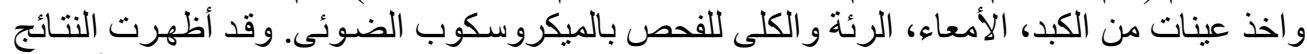

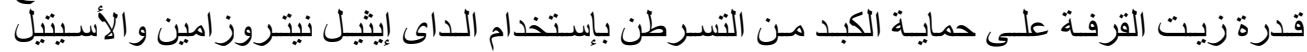

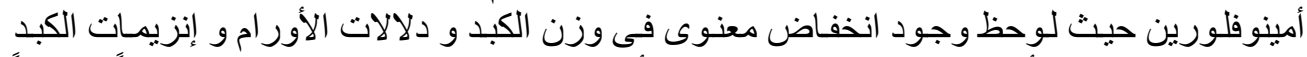

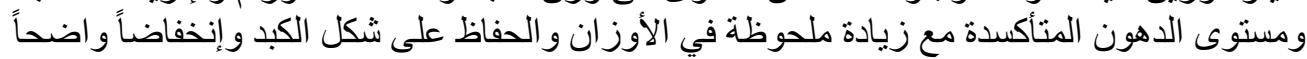

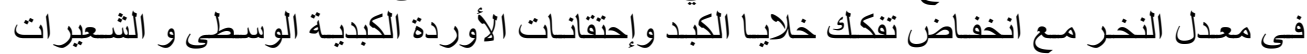

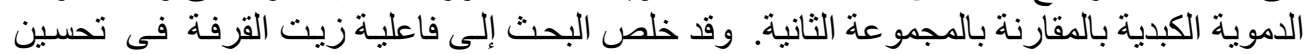

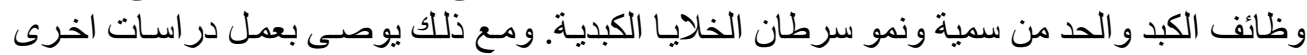
متقدمة قبل استخدامهما مع العلاجات الاخرى المتاحة لسرطان الخدان الخلايا الكبدية. 